\title{
Beam Size Measurement of the Stored Electron Beam at the APS Storage Ring Using Zone Plate Optics and Undulator Radiation
}

\author{
Zhonghou Cai, Barry Lai, Wenbing Yun, Efim Gluskin, Dan Legnini, \\ Peter Ilinski, Emil Trakhtenberg, Shenglan Xu, \\ William Rodrigues, and Heung-Rae Lee
}

Advanced Photon Source, Argonne National Laboratory, Argonne, IL 60439

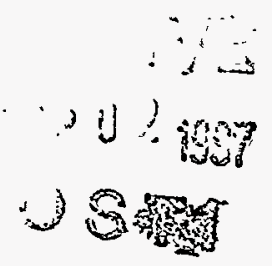

\begin{abstract}
Beam sizes of the stored electron beam at the Advanced Photon Source storage ring were measured using zoneplate optics and undulator radiation. A gold Fresnel zone plate ( $3.5 \mu \mathrm{m}$ thick) located 33.9 meters from the $x$-ray source focused radiation of $18 \mathrm{keV}$, selected by a cryogenically cooled $\mathrm{Si}(111)$ crystal in horizontal deflection, and formed a source image in a transverse plane $2.41 \mathrm{~m}$ downstream. The sizes of the source image were determined from measured intensity profiles of $x$-ray fluorescence from a smooth nickel edge ( $1.5 \mu \mathrm{m}$ thick), fabricated using a lithographic technique, while the nickel edge was scanned across over the beam in the transverse plane. The measured vertical and horizontal sizes of the electron beam were $60 \pm 4.3 \mu \mathrm{m}$ and $300 \pm 13 \mu \mathrm{m}$, respectively, in reasonable agreement with the expected values.
\end{abstract}

\section{INTRODUCTION}

As the Advanced Photon Source (APS) begins operation, beam emittance measurements become an essential part of the commissioning process to diagnose the performance of the storage ring and insertion devices. In the center of the straight section, beam size and divergence are determined by the natural beam emittance, coupling between vertical and horizontal emittances, and the $\beta$ functions characterizing the magnet lattice in the storage ring (1). Beam size measurements provide useful information about those values. When diagnosing undulator radiation, beam size is usually needed to convert the results obtained from absolute flux measurement to beam brilliance (2). Even after the APS enters the operational mode, beam size information will be desired for the experiments involving $\mathrm{x}$-ray focusing.

Usually, the divergence of the photon beam can be determined accurately by measuring the photon beam size $\sigma$ at a distance $D$ from the source. That is

$$
\sigma^{2}=D^{2} \sigma_{s}^{2}+\sigma_{s}^{2},
$$

where $\sigma_{s}$ and $\sigma_{s}$, refer to the size and the divergence of the photon source, respectively. When the measurement takes place at a large $D, \sigma$ can be much larger than $\sigma_{s}$. The uncertainty of the measurement can be well managed and limited at a relatively small value. For undulator radiation, however, the inherent divergence of the photon beam, which is comparable with the divergence of the particle beam in APS storage ring, prevents us from accurately determining the divergence of the particle beam. As such, accurate measurements of beam size become an important issue in the diagnostics of beam emittance. Especially because our diagnostics were focused not only on the particle beam but also on the undulator photon beams, the technique selected has to accommodate the requirement for portability of the diagnostic equipment. In the next section, a comparison of pinhole optics and zone-plate optics is given to explain our choice of zone-plate optics.

\section{ZONE-PLATE OPTICS AND PINHOLE OPTICS}

A phase zone plate is a diffractive focusing device made of a number of concentric circular zones. The thicknesses of the zones are determined so that a phase shift of $\pi$ is obtained for a given radiation wavelength. The focal length of the zone plate 


\section{DISCLAMMER}

Portions of this document may be illegible in electronic image produets. Images are produced from the best available original document. 


\section{DISCLAIMER}

This report was prepared as an account of work sponsored by an agency of the United States Government. Neither the United States Government nor any agency thereof, nor any of their employees, make any warranty, express or implied, or assumes any legal liability or responsibility for the accuracy, completeness, or usefulness of any information, apparatus, product, or process disclosed, or represents that its use would not infringe privately owned rights. Reference herein to any specific commercial product, proces, or service by trade name, trademark, manufacturer, or otherwise does not necessarily constitute or imply its endorsement, recommendation, or favoring by the United States Government or any agency thereof. The views and opinions of authors expressed herein do not necessarily state or reflect those of the United States Government or any agency thereof. 
depends on the refractive index of the zone material, the wavelength of the radiation, and the Fresnel half-period radii. Zone plates work like thin lenses, so that the thin lens formula,

$$
\begin{gathered}
\frac{1}{Z_{1}}+\frac{1}{Z_{2}-Z_{1}}=\frac{1}{f}, \\
M=\frac{Z_{2}-Z_{1}}{Z_{1}},
\end{gathered}
$$

applies for the formation of the source image (3), where $Z_{1}$ is the source-zone-plate distance, $Z_{2}$ is the source-image-plane distance, $f$ is the focal length of the zone plate, and $M$ is the magnification factor of the imaging system. A zone plate has a diffraction limit to its transverse resolution similar to that calculated by Rayleigh for a thin lens, which turns out to be about $122 \%$ of the outermost zone width of the zone plates (3). With currently available manufacturing techniques for hard $x$ ray zone plates, the diffraction-limited resolution can be smaller than $0.2 \mu \mathrm{m}$.

Pinhole imaging has been used recently for source-size measurements during commissioning of several third-generation synchrotron radiation sources (47). The pinhole optics describing the relation between the measured size of the source image on the screen $\sigma$ and the source size $\sigma_{s}$ can be expressed as (7)

$$
\sigma^{2}=\left(\frac{Z_{2}-Z_{1}}{Z_{1}}\right)^{2} \sigma_{s}^{2}+\left(\frac{Z_{2}}{Z_{1}}\right)^{2} \sigma_{p}^{2} \text {, }
$$

where $\sigma_{p}$ refers to the rms size of the pinhole, $Z_{1}$ and $Z_{2}$ are, respectively, the distances of the pinhole and the image screen from the source. The first term in Eq. (4) represents the size of the source image, and the second term reflects the size of the pinhole projected on the screen. Note that the image broadening given by the second term is evaluated with a point-source approximation. It is valid when $\sigma_{s} \ll Z_{1} \sigma_{s}$. The Fraunhofer diffraction of x-rays from a pinhole results in an angular spreading of the $x$-ray beam and, thus, a broadening of the source image. The rms image broadening due to $x$-ray diffraction is about $\left(Z_{2}-Z_{1}\right) \lambda / 7 \sigma_{p}$, where $\lambda$ is the wavelength of the $\mathrm{x}$-rays (8).

For the APS, the radiation characteristics are dominated by the emittance of the stored particle beam. Given a natural beam emittance of $8.2 \mathrm{~nm}$-rad, a horizontal $\beta$ function of $14.2 \mathrm{~m}$, and a vertical $\beta$ function of $10 \mathrm{~m}$, the horizontal and vertical sizes of the electron beam in the storage ring would be $325 \mu \mathrm{m}$ and $86 \mu \mathrm{m}$ for a coupling constant of $10 \%$, and 333 $\mu \mathrm{m}$ and $62 \mu \mathrm{m}$ for a coupling constant of $5 \%$ (9). The vertical beam size becomes smaller as the coupling constant decreases. Consider an undulator radiation source with rms size of $60 \mu \mathrm{m}$. For measurements using pinhole optics, if a pinhole of $5 \mu \mathrm{m}$ $\left(\sigma_{p}\right)$ is placed 10 meters from the source and the imaging screen is located 10 meters downstream, the rms sizes of the source image, the projection of the pinhole, and the broadening due to diffraction are, respectively, $60 \mu \mathrm{m}, 10 \mu \mathrm{m}$, and $35 \mu \mathrm{m}$ (for $10 \mathrm{keV} \mathrm{x}$-rays). The size of the image on the screen is a convolution of the three quantities. Note that the diffraction broadening is about $60 \%$ of the size of the source image. The uncertainty in pinhole size will generate a significant error when deconvolution of the image size from the diffraction broadening and the finite size of the pinhole is carried out. This error becomes severe when the source size is small like that of the APS. In order to reduce the diffraction effect, one can either employ high energy radiation or make the distance $Z_{2}-Z_{1}$ small. However, small pinholes for high energy $x$-rays are difficult to manufacture, and one would also reduce the source-pinhole distance $Z_{1}$ if $Z_{2}-Z_{1}$ is reduced so that a magnification factor required for accurate measurement can be maintained. At the APS, this approach means that the measurement has to be conducted in the front end and a dedicated beamline is required for beam diagnostics. For the diagnostics of insertion devices, this approach is practically impossible because each device has to be installed in the section of the dedicated beamline once there is a need for the device to be diagnosed.

For measurements using zone-plate optics, if a zone plate with a focal length of $2.5 \mathrm{~m}$ is located 30 meters from the source, the rms size of the source image on the image plane will be $5 \mu \mathrm{m}$, and the diffraction-limited transverse resolution $(0.2 \mu \mathrm{m})$ is only a small fraction of the source-image size. Because the diffraction-limited resolution is small, the accuracy of the source size measurement is not sensitive to the source-zone-plate distance. In other words, the measurements can take place in stations located at various distances from undulator sources. We have developed a technique for source size measurement using $x$-ray zone-plate optics, in which the source was imaged at the imaging plane of the zone plate, and the image size was measured by scanning a nickel thin edge across the beam in the imaging plane and measuring its fluorescence. 


\section{EXPERIMENTAL SETUP}

The experiment was performed in the first optical enclosure (FOE) of the 2-ID beamline of the APS. The $x$-ray source was the synchrotron radiation generated from undulator A (9). Fig. 1 is a schematic of the experimental setup. In order to reduce the thermal load on the monochromator, the radiation extracted from the storage ring was, first, filtered with a set of filters consisting of $500 \mu \mathrm{m}$ carbon, $150 \mu \mathrm{m}$ diamond, and $500 \mu \mathrm{m}$ beryllium. Then, the power of the undulator radiation was further reduced substantially by a water-cooled grazing incidence conical pinhole (copper) with the exit hole $800 \mu \mathrm{m}$ in

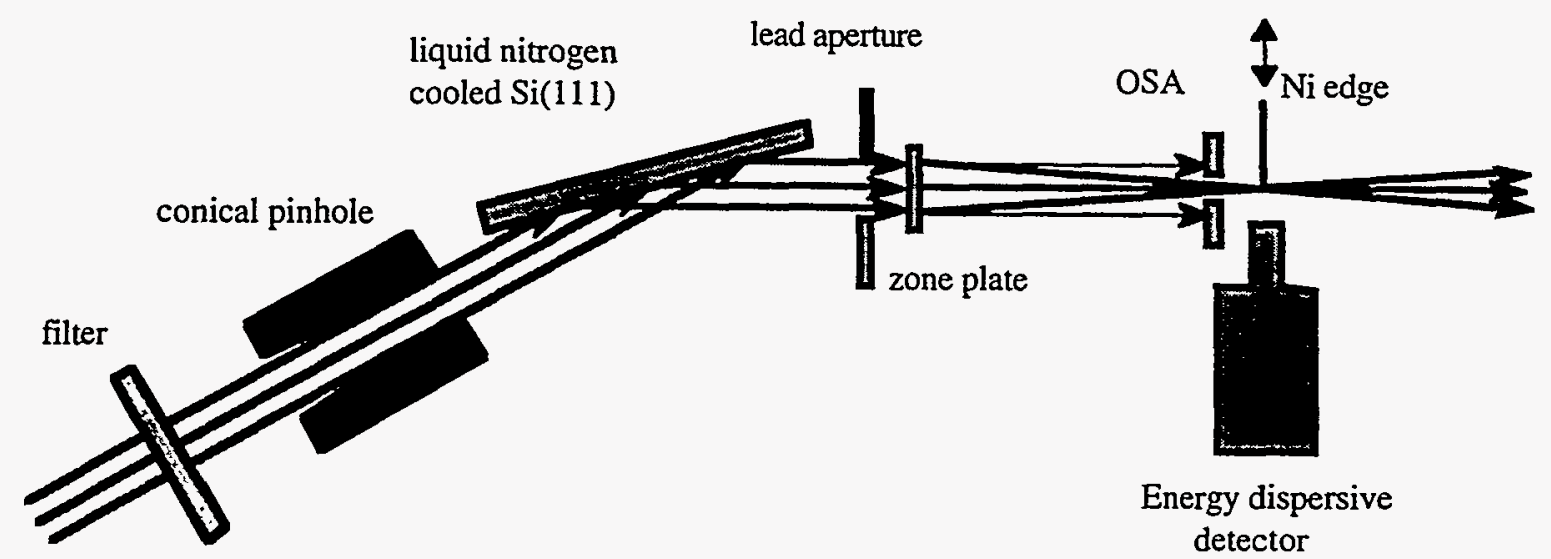

$\mathrm{x}$-ray beam

FIGURE 1. Schematic illustration of the experimental setup.

diameter. X-rays of $18 \mathrm{keV}$ were selected by a cryogenically cooled Si(111) crystal in horizontal deflection. The vacuum chamber for the monochromator was sealed with two $150-\mu \mathrm{m}$-thick beryllium windows capable of tuning the $\mathrm{x}$-ray energy from 7 to $30 \mathrm{keV}$. The monochromatic beam was apertured to a size of $600 \mu \mathrm{m}$ before it illuminated the zone plate. A gold zone plate of $400 \mu \mathrm{m}$ in diameter ( $3.5 \mu \mathrm{m}$ thick) with a focal length of $2.25 \mathrm{~m}$ was located $33.89 \mathrm{~m}$ from the center of the straight section, and thus the $\mathrm{x}$-ray source image was formed in a transverse plane $2.41 \mathrm{~m}$ downstream from the zone plate. A platinum order-sorting aperture (OSA) of size $30 \mu \mathrm{m}$ was placed $100 \mathrm{~mm}$ upstream from the image plane to increase the contrast of the source image. An energy-dispersive detector (Si(Li), EG\&G ORTEC SLP 06165PS) was located close to the imaging plane pointing in a direction normal to the $\mathrm{x}$-ray beam to measure the $\mathrm{x}$-ray fluorescence generated from a nickel thin

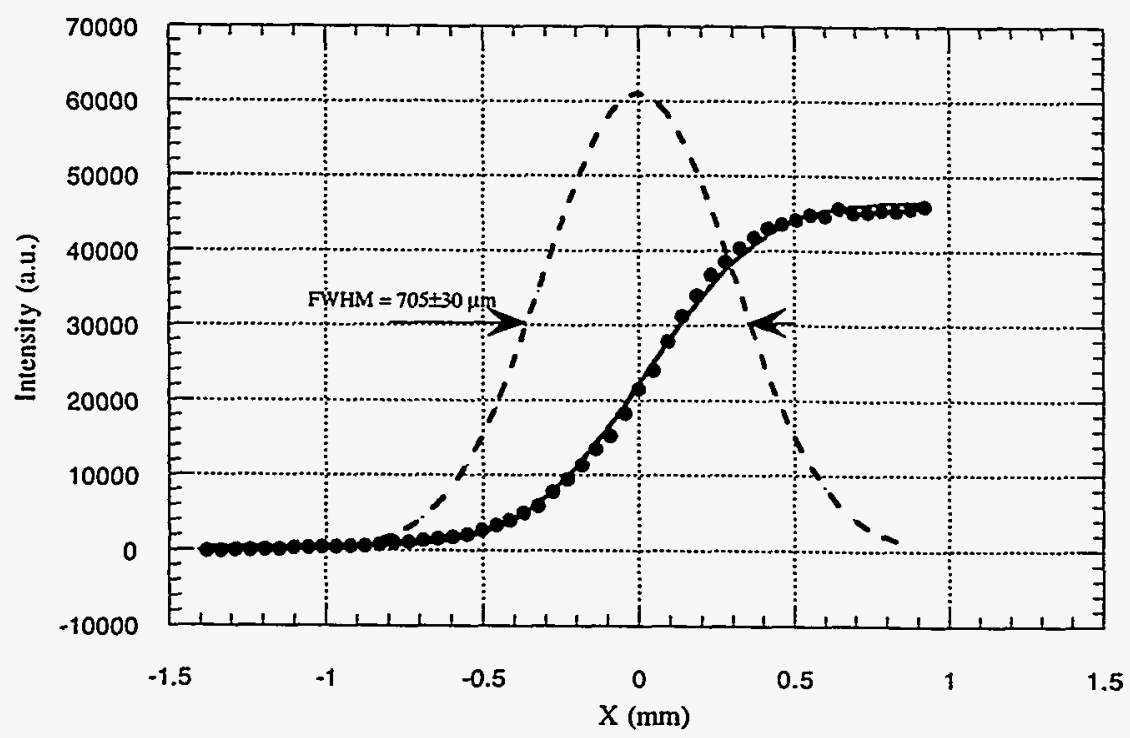

FIGURE 2. Fluorescence intensity profile of a horizontal knife-edge scan (dots). The solid line is the fitted curve to the experimental data, and the dashed line is the derivative of the fitted curve. The horizontal axis reflects the coordinates at the source location. 
edge ( $\mathrm{K} \alpha$ line) placed in the imaging plane. The beam sizes were determined from the fluorescence intensity profiles measured when the nickel edge was being scanned across the beam.

The FOE was chosen because the experimental stations downstream had not yet been commissioned when the measurements were made. The measurements were taken in the presence of a high radiation background because the detector had to be in the same station where radiation scattering components, such as the pinhole and the monochromator, were installed. Therefore, the conical pinhole was placed in a helium enclosure, and both the pinhole enclosure and the monochromator vacuum chamber were carefully shielded with lead and steel.

\section{RESULTS}

All measurements were performed with a beam of $7 \mathrm{GeV}$ and less than $5 \mathrm{~mA}$. For the natural emittance and the emittamce coupling of the beam in the storage ring, the $\beta$ functions at the center of the straight section, and the experiment configuration, the source image on the image plane would be $6 \mu \mathrm{m}$ vertically and $23 \mu \mathrm{m}$ horizontally. The performance of the crystal monochromator and the energy bandwidth of the $\mathrm{x}$-ray beam intercepted by the zone plate are critical to the accuracy of the measurement.

Main concerns about the performance of the crystal monochromator arise from the possibility of distortion in the atomic planes of the crystal due to the high power of the undulator radiation that would shift the virtual source away from the point of the real source. The undulator gap was set at $14.8 \mathrm{~mm}$ so that its third harmonic peaked at $18 \mathrm{keV}$. With a $800 \mu \mathrm{m}$ aperture located at $33 \mathrm{~m}$, the total incident power of the undulator radiation was about $5 \mathrm{~W}$ with a current of $5 \mathrm{~mA}$. The high thermal conductivity of the silicon at liquid-nitrogen temperature should be able to maintain the atomic planes without distortion. The width of the rocking curve of the monochromator measured with an analyzing crystal ( $\mathrm{Si}(111))$ at beam current of $20 \mathrm{~mA}$ was found to be the same as the ideal value. Horizontal deflection takes advantage of less stringent requirements in slope error for avoiding image distortion in the vertical direction along which a small image size is expected.

Because of single bounce, the monochromatic nature of the $x$-ray beam is determined by the intrinsic energy bandwidth of the crystal and the angular acceptance of the zone plate. Given the distance of the zone plate from the source, we found that the energy bandwidth of the $\mathrm{x}$-ray beam picked up by the zone plate was about $5 \mathrm{eV}$, and image broadening due to the bandwidth can be neglected.

We show the measured nickel $(K \alpha)$ fluorescence intensity profile of a horizontal knife-edge scan in Figure 2. The experimental data were fitted with an error function and a uniform background was assumed. The horizontal intensity profile of the source image was then obtained by taking the derivative over the fitted fluorescence intensity profile. In the figure, the profiles are plotted versus the product of the dimension at the image plane with the demagnification factor of the zone-plate imaging system, representing the dimension at the source point. The beam intensity profile gives a horizontal source size of $705 \mu \mathrm{m}$ at the full width half maximum, or $300 \mu \mathrm{m}$ in rms size.

Figure 3 displays a similar fluorescence intensity profile for the vertical knife-edge scan. It provides a vertical source size of $141 \mu \mathrm{m}$ at the full width half maximum, or $60 \mu \mathrm{m}$ in rms size. The distance between the zone plate and the nickel edge was optimized so that the most narrow intensity profile of the source image was obtained. From the results of source-

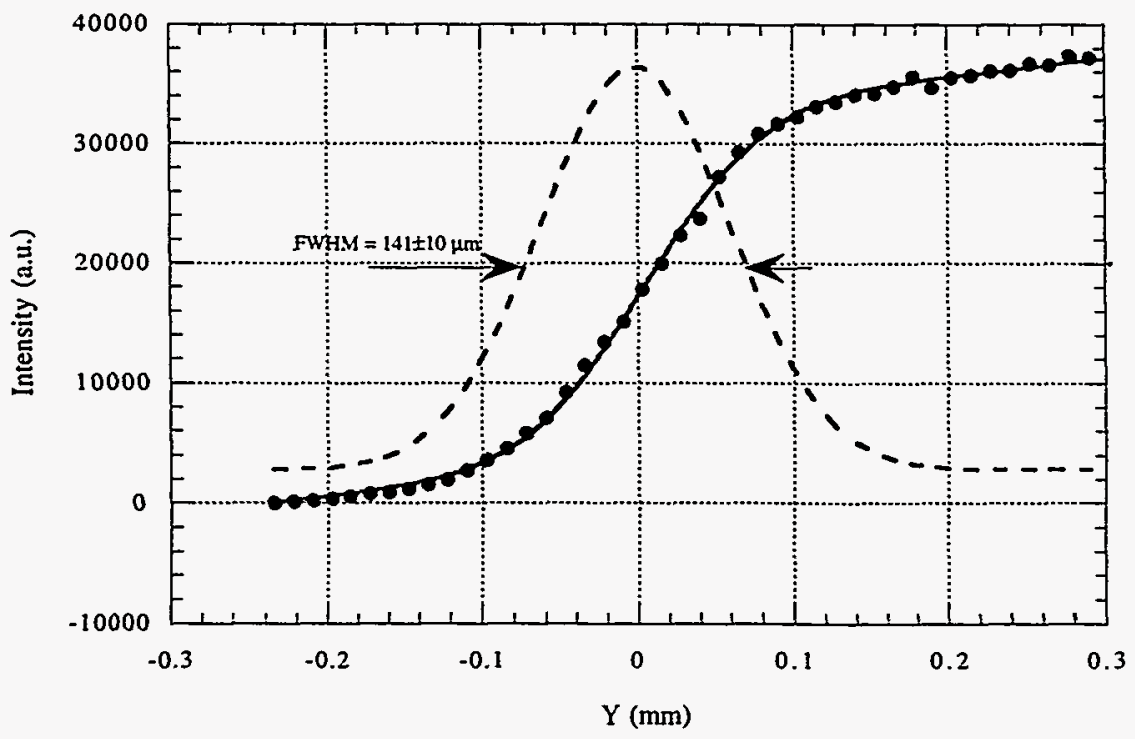

FIGURE 3. Fluorescence intensity profile of a vertical knife-edge scan (dots). The solid line is the fitted curve to the experimental data, and the dashed line is the derivative of the fitted curve. The horizontal axis uses the coordinates at the source location. 
size measurements and beam-divergence measurements (2), we obtained a horizontal beam emittance of $7.5 \mathrm{~nm}-\mathrm{rad}$ and a vertical beam emittance of $0.32 \mathrm{~nm}$-rad, and, thus, the coupling constant is 0.043 .

\section{DISCUSSION}

The accuracy of the measurement depends on the accuracies of the distances of the zone plate and the knife edge from the source, the quality of the $\mathrm{Ni}$ thin edge, and the mechanical stability of the imaging system. The Ni thin edge was manufactured using a lithographic technique and was checked by a scanning electron microscope (SEM) before the experiment. Figure 4 is a photograph of the SEM backscattering image of the nickel edge used in the experiment. The smoothness of the edge is better than $0.1 \mu \mathrm{m}$. In order to evaluate the performance of the thin edge and the stability of the mechanical system, the same nickel edge was used to measure the spot size of an x-ray beam focused with a 6-cm-focal-length zone plate using the same setup and the same source-zone-plate distance. A $0.2 \mu \mathrm{m}$ beam spot size (rms) was obtained, indicating the roughness of the nickel edge and the instability of the system almost have no effect on the source size measurement.

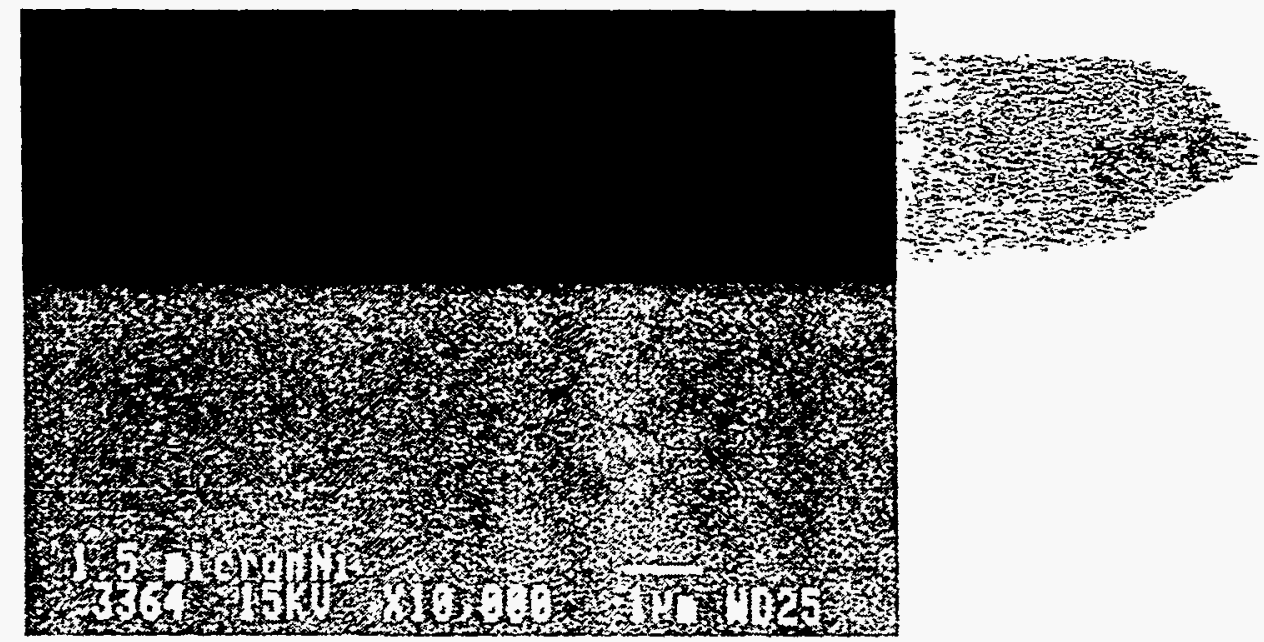

FIGURE 4. SEM backscattering image of the nickel thin edge.

In a summary, a zone-plate-optics-based, portable, and flexible beam diagnostics instrument for undulator source size (emittance) measurement has been developed. The instrument has been used for size measurement of the particle beam in the APS storage ring and for undulator photon beam diagnostics. Measured results were in reasonable agreement with expected values.

\section{ACKNOWLEDGMENTS}

Work was supported by the U.S. Department of Energy, BES. Material Sciences, under contract No. W-31-109-Eng-38.

\section{REFERENCES}

1. Sands, M., The Physics of Electron Storage Ring. Proceedings of the Int. School 'Enrico Fermi', 1971, Vol. 46, p. 258.

2. Cai, Z., Dejus, R., Gluskin, E., Ilinski. P., Lai, B., Yun, W., and et al, Rev. Sci. Instrum., 67 (1996) CD ROM.

3. Klein, M. V., Optics, Wiley, New York, 1970.

4. Mills, D., Viccaro, P., Merlini, A., and Shen, Q., Nucl. Instrum. \& Meth. Phys. Res. A291, 481 (1990).

5. Elleaume, P., Fortgang, C., Penel, C.. and Tarazona, E., J. Synchrotron Rad. 2, 209 (1995).

6. Cai, Z., Lai, B., Yun, W., Gluskin, E., Dejus, R., and Ilinski, P., Rev. Sci. Instrum., 66, 1859(1995).

7. Cai, Z., Lai, B., Yun, W., Gluskin, E.. Legnini, D., Illinski, P., and et al, Rev. Sci. Instrum., 67 (1996) CD ROM.

8. Born M. and Wolf, E., Principles of Optics, Pergamon, Oxford, 1980.

9. Dejus, R., Lai, B., Moog, E., and Gluskin, E., Argonne National Laboratory Report, ANL/APS/TB-17 (1994). 